\title{
Brief Communication: The recent seismic activity in Central Greece in 2013 and its precursory electric signals in terms of criticality
}

\author{
E. Dologlou \\ Solid State Section, Department of Physics, University of Athens, Panepistimiopolis, Zografou 15784 Athens, Greece \\ Correspondence to: E. Dologlou (edologl@phys.uoa.gr)
}

Received: 23 September 2013 - Revised: 17 December 2013 - Accepted: 17 December 2013 - Published: 29 January 2014

\begin{abstract}
Here, we check the obedience of new data, derived from the $M_{\mathrm{w}}=5.4$ earthquake on 7 August 2013 in Central Greece, to a previously found power law relation by the author between the stress drop of an earthquake and the lead time of its precursory seismic electric signal (SES). An exponent value $\alpha=0.329$ has been found which is in excellent agreement with previous ones reported in a series of articles by the author. This value falls in the range of critical exponents suggested by various models for fracture and is very close to a reported one which interconnects the amplitude of the SES and the magnitude of the impending earthquake. The stability of this exponent confirms the credibility of the above-mentioned power law and probably implies that real physical dynamic processes evolving to criticality are present in the pre-focal area when the SES is emitted.
\end{abstract}

\section{Introduction}

The concept that the preparation processes of large earthquakes can be better understood in terms of statistical physics of a critical phase transition culminating in a cataclysmic event, is widely adopted by many investigators (Andersen et al., 1997; Keilis-Borok, 1990; Rundle et al., 2000).

Fracture in heterogeneous materials, such as the highly fragmented Earth's crust, could be viewed as a critical phenomenon and has been a field of active study in recent years (Lamaignère et al., 1996; Sornette and Sornette, 1990). The critical point hypothesis suggests that large earthquakes only occur when the pre-focal area is in critical stage. This implies that the pre-seismic region behaves as a system at the boundary between order and disorder and is characterized by fractal geometry (Rong et al., 2012) including both origins of self-similarity (i.e. process memory and process increments' infinite variance, e.g. see Varotsos et al., 2006; Sarlis et al., 2010), strong long-range correlations between different parts, and non-linear dynamic processes (Telesca et al., 2005).

The investigation of possible earthquake precursors is one of the top targets in Earth science today. Chief among them are the electromagnetic anomalies which appear in a wide frequency band from $\mathrm{DC}$ to $\mathrm{MHz}$ during the last preparatory stage of the main shock (Gokhberg et al., 1982; Varotsos and Alexopoulos, 1984a, b; Hayakawa et al., 2000; Telesca et al., 2001; Nagao et al., 2002; Huang, 2011a; Xu et al., 2013). Here, we focus on DC-ULF electric signals, termed seismic electric signals (SES), which are recognized as transient lowfrequency $(\leq 1 \mathrm{~Hz})$ variations of the Earth's telluric field and have been found to precede large earthquakes in Greece and Japan (Varotsos and Alexopoulos, 1984a, b; Varotsos et al., 1993; Orihara et al., 2012). They are emitted from the prefocal area when the tectonic stress reaches a critical value which also signals the entrance of the region into a critical stage.

A plausible model for the SES generation which is based on the existence of point defects in solids (Varotsos and Alexopoulos, 1977) is as follows: rocks in the Earth's crust contain various ionic materials with lattice defects forming electric dipoles with nearby vacancies. When the tectonic stress in the candidate focal area reaches a critical value, a cooperative change in these dipoles' orientation results in a transient current which constitutes the SES. Details of this model can be found elsewhere (Varotsos et al., 1998). Other possible generation mechanisms are the piezoelectric effect (Huang, 2002, 2011b) and the electrokinetic effect (Ren et al., 2012). A series of such successive signals forms a SES activity (Varotsos and Lazaridou, 1991). It has been found (Varotsos et al., 2002; Abe et al., 2005) that the SES activities exhibit scale invariant structure (power law) (Varotsos et al., 2003) which is consistent with the criticality concept of the SES generation model mentioned above. 
Two of the most fundamental features of these SES signals are the lead time and the selectivity. The lead time, $\Delta t$, is the time difference between the detection of the SES and the occurrence of the associated earthquake, and varies from some hours (for a single signal) to a few months (for SES activity) (Varotsos and Alexopoulos, 1984a, b). Recently, the introduction of a new time domain, termed natural time (Varotsos et al., 2002), allows the identification of the time of the impending earthquake (Sarlis et al., 2008) with accuracy of the order of one week. The selectivity effect is the ability of a SES station to be sensitive to some specific seismic areas and insensitive to some others even at closer distances (Varotsos and Lazaridou, 1991; Varotsos et al., 1993). There is some evidence of SES selectivity from the results of analogue experiments (Huang and Ikeya, 1998, 1999) and numerical simulations (Huang and Lin, 2010a, b).

A series of recent articles (Dologlou, 2008, 2009, 2010, 2012,2013 ) showed an interconnection between dynamic parameters of large earthquakes and features of their precursory SES in terms of criticality. Precisely, a power law relation with an exponent falling in the range of critical values for fracture has been found between the lead time of the SES and the stress drop of the earthquake. The objective of the present work is to check the obedience of new data to this power law relation. It is worth noting that the same power law should hold between the stress drop of major earthquakes $(M>6.5)$ and the precursory magnetic field variations accompanying SES activities (Skordas et al., 2010).

\section{Data and discussion}

Here, we focus on the update of the power law between the lead time of the SES and the stress drop of the earthquake, by introducing the most recent data from 9 January 2013 to 23 September 2013 (see Dologlou, 2013 - Table 2). Two events with $M_{\mathrm{w}}>5.0$ have been reported in the region (36$41)^{\circ} \mathrm{N}(19-26)^{\circ} \mathrm{E}$ (EMSC, 2013) during this period. First, the $M_{\mathrm{w}}=5.4$ (USGS) earthquake, which occurred on $7 \mathrm{Au}-$ gust 2013 at 09:56:04 (UTC) with an epicentre $\left(38.71^{\circ} \mathrm{N}\right.$ $22.66^{\circ} \mathrm{E}$ ) (big blue star in Fig. 1). This is also the largest event that has taken place in the broader area during the last ten years. Second, the $M_{\mathrm{w}}=5.3$ (USGS) earthquake on 16 September 2013 at 15:01:14 (UTC) with an epicentre $\left(38.70^{\circ} \mathrm{N} 22.73^{\circ} \mathrm{E}\right)$ (small blue star in Fig. 1), falling in the aftershock area of the first one.

The CMT-Harvard fault plane solution of the $M_{\mathrm{w}}=5.4$ main shock (USGS, 2013) is of normal type (Fig. 1 beach ball), since the epicentre lies in the basin between Mt. Kallidromon and Mt. Parnassos, Central Greece, on the Kalidromon-Atalanti-Martino Fault Zone (KAMFZ) (Fig. 1 - dashed lines), a region well known for its active and fast extension. This regime is vividly characterized by a series of subparallel graben oriented roughly WNW-ESE accommodating mainly normal movement (Pantosti et al., 2001).

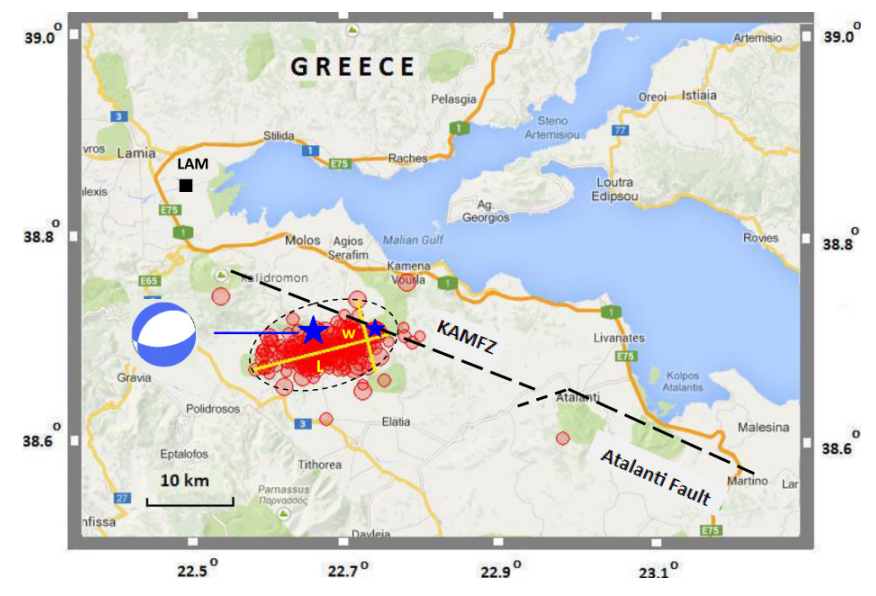

Fig. 1. Map of Central Greece, with the distribution (forming a red cluster) of aftershocks with $M \geq 2.3$ reported by EMSC, for the period from 7 August 2013 to 17 September 2013. Dashed ellipse highlights the location of the aftershock area with dimensions $W$ and $L$, while big and small blue stars denote the epicentres of the main shock and the latest $M_{\mathrm{W}}=5.3$ event, respectively. Black square depicts the location of the SES station, LAM. The Kallidromon-Atalanti-Martino fault zone (KAMFZ) as well as the Atalanti fault are represented by dashed lines. USGS-reported focal mechanism is shown as a beach ball and a lower hemisphere projection is used with blue and white quadrants for compression and dilatation.

A rich aftershock sequence of more than 300 events $(M>$ 2.3), forming a well-defined cluster (Fig. 1 dashed ellipse) of dimensions $L \times W$, followed the main shock. All aftershocks are taken from the European Mediterranean Seismological Centre (EMSC) for the period from 7 August 2013 to 23 September 2013 (EMSC, http://www.emsc-csem.org).

This $M_{\mathrm{w}}=5.4$ earthquake was preceded by a SES activity recorded at Lamia station (LAM), close to Lamia, Central Greece (Fig. 1 - solid square), from 31 March to 11 April 2013 (this is the longest SES duration ever observed in Greece) with a lead time $\Delta t=129$ days (Varotsos et al., 2013).

In order to calculate the Brune stress drop we used the formula of Hanks and Wyss (1972):

$\Delta \sigma_{B}=0.44 M_{\mathrm{o}} / r^{3}$,

where $M_{\mathrm{o}}$ is the seismic moment and $r$ the radius for a circular fault. The estimation of the radius $r$, was obtained by the application of the aftershock area technique (Kiratzi et al., 1991) as it is described in detail by Dologlou (2009). The aftershock area $S=L \times W$ in $\mathrm{km}^{2}$, with length $L$ and width $W$, is clearly defined from the distribution of the aftershocks (Fig. 1 - red cluster). The radius $r$ is calculated from the equation $S=\pi r^{2}$. The values $L$ and $W$ along with their error range and mean values are presented in Table 1. According to USGS the seismic moment of the main shock is $M_{\mathrm{o}}=1.2 \times 10^{17} \mathrm{Nm}($ Table 1$)$. 
Table 1. The USGS-reported date and magnitude of the main shock along with its seismic moment, range of values in the dimensions of the aftershock areas $L$ and $W$, the corresponding calculated values for $\Delta \sigma_{B}$, the critical exponent $\alpha$ and the lead time $\Delta t$. Mean values are also given.

\begin{tabular}{llcccllll}
\hline No. & Date & $M_{\mathrm{W}}$ & $\begin{array}{c}M_{\mathrm{O}} \\
\left(10^{24} \text { dyn.cm }\right)\end{array}$ & $\begin{array}{c}L \\
(\mathrm{~km})\end{array}$ & $\begin{array}{l}W \\
(\mathrm{~km})\end{array}$ & $\begin{array}{l}\Delta \sigma_{B} \\
(\mathrm{bars})\end{array}$ & $\alpha$ & $\begin{array}{l}\Delta t \\
\text { days }\end{array}$ \\
\hline 23 & 7 Aug 2013 & 5.4 & 1.2 & $15-17$ & $8.5-9.5$ & $2.04-1.43$ & $0.324-0.335$ & 129 \\
& Mean value & & & 16 & 9 & 1.70 & $0.329 \pm 0.010$ & \\
\hline
\end{tabular}

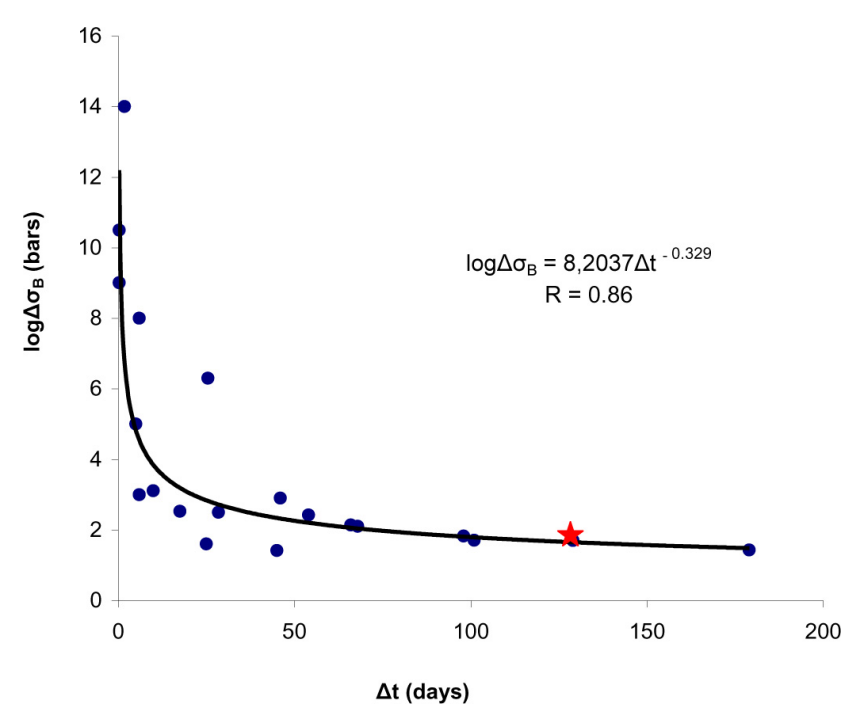

Fig. 2. The plot of the power law between the stress drop of the earthquakes and the lead time of SES. A red star corresponds to the new data (Table 1, no. 23), while solid circles refer to the data sets reported in Table 2 of Dologlou (2013). The new derived power law relation along with its correlation coefficient $\mathrm{R}$ and the exponent $\alpha=0.329$ are displayed on the top of the diagram.

The introduction now of these values into the latest reported (Dologlou, 2013) power law relation $\Delta \sigma_{B}=$ $8.213 \Delta t^{-0.330}$, which is under a continuous updating process by the author, leads to an exponent $\alpha=0.329 \pm 0.010$ with a correlation coefficient $R=0.86$ (see Fig. 2 and Table 1). All the calculated values, i.e. the dimensions of the aftershock area, the stress drop and the critical exponent along with their error range and their mean values are also given in Table 1. We remark that events nos. 11, 13, and 15 (Table 2 of Dologlou, 2013) are always excluded from this relation for reasons explained in detail elsewhere (Dologlou, 2008, 2009). This new exponent 0.329 is in excellent agreement with the previously found one (i.e. 0.330 ) by Dologlou (2013), and falls within the range of the values of critical exponents suggested by various models for fracture. Furthermore, it is very close to the reported exponent by Varotsos and Alexopoulos (1984a), which interconnects the amplitude $E$ of the SES and the magnitude $M$ of the impending earthquake according to the following power law relation that is reminiscent of the theory of critical phenomena

$\log E=a M+b$,

where $E=\Delta V / L$ with $\Delta V$ the potential difference between two points on the ground at a distance $L$, measured by two buried electrodes, $a \approx 0.3-0.4$ and $b$ is a site constant depending on the geoelectrical structure around the station.

The remarkable stability of this exponent, confirmed by a large amount of data (see Table 1 of Dologlou, 2013), reflects the reliability of the above power law and probably implies that the SES emission marks the entrance of the pre-focal region into a critical stage where non-linear dynamic processes and long-range correlations prevail. It is worth noting that when inappropriate data are inserted into this power law, the value of the exponent is significantly violated (see Dologlou, 2013, 2012).

Nature has its own rules which sometimes deviate from the expected behaviour, as in the case of the under study aftershock sequence. Thus, we have to mention that the $M_{\mathrm{w}}=5.3$ (USGS) earthquake that occurred on 16 September 2013 with an epicentre falling in the margins of the aftershock area (Fig. 1 - small blue star) was followed by numerous smaller events in which the spatial distribution does not alter the dimensions of the aftershock area depicted in Fig. 1. However, this eastward migration of the epicentre of this new $M_{\mathrm{w}}=5.3$ earthquake (Fig. $1-$ small blue star) might trigger the highly potential Atalanti fault (Fig. 1 - dashed line). On the other hand, the unusually long duration of the SES activity recorded at LAM station could be considered as an underlying hint that the whole seismic activity in the broader area is still in progress. Here, we restrict ourselves to the present situation and data and we carefully follow with great interest the evolution of the phenomenon.

\section{Conclusions}

The update of a previously found power law relation between the stress drop of an earthquake and the lead time of its associated SES, by inserting new data from the $M_{\mathrm{w}}=5.4$ earthquake on 7 August 2013 in Central Greece, led to an exponent value $\alpha=0.329$ which is in excellent agreement with the reported ones by the author. This value falls in the range of critical exponents suggested by various models for fracture and is very close to the one which interconnects the 
amplitude of the SES and the magnitude of the impending earthquake. The stability of this exponent confirms the credibility of the above power law and probably implies that real physical dynamic processes evolving to criticality are present in the pre-focal area when the SES is emitted.

Edited by: L. Telesca

Reviewed by: two anonymous referees

\section{References}

Abe, S., Sarlis, N. V., Skordas, E. S., Tanaka, H. K., and Varotsos, P. A.: Origin of the usefulness of the natural time representation of complex time series, Phys. Rev. Lett., 94, 170601, doi:10.1103/PhysRevLett.94.170601, 2005.

Andersen, J. V., Sornette, D., and Leung, K.-T.: Tri-critical behavior in rupture induced by disorder, Phys. Rev. Lett., 78, 2140-2143, 1997.

Dologlou, E.: Possible relationship between Seismic Electric Signals (SES) lead time and earthquake stress drop, P. Jpn. Acad. B-Phys., 84, 117-122, doi:10.2183/pjab.84.117, 2008.

Dologlou, E.: Power law relationship between parameters of earthquakes and precursory electrical phenomena revisited, Nat. Hazards Earth Syst. Sci., 9, 17-23, doi:10.5194/nhess-9-17-2009, 2009.

Dologlou, E.: Power law relationship between parameters of earthquakes and precursory electrical phenomena revisited II, Nat. Hazards Earth Syst. Sci., 10, 1403-1409, doi:10.5194/nhess-101403-2010, 2010.

Dologlou, E.: Stability of a power law relation between characteristics of earthquakes and electric precursors, Nat. Hazards Earth Syst. Sci., 12, 1783-1787, doi:10.5194/nhess-12-17832012, 2012.

Dologlou, E.: Features of criticality in precursory seismic electric signals and earthquakes in Greece, Nonlin. Processes Geophys., 20, 411-416, doi:10.5194/npg-20-411-2013, 2013.

EMSC (The European-Mediterranean Seismological Centre): http: //www.emsc-csem.org/Earthquake/?filter=yes, last access: 2013.

Gokhberg, M., Morgunov, V., and Tomizawa, I.: Experimental measurements of electromagnetic emissions possibly related to earthquake in Japan, J. Geophys. Res., 87, 7824-7828, 1982.

Hanks, T. and Wyss, M.: The use of body wave spectra in the determination of seismic source parameters, B. Seismol. Soc. Am., 62, 561-589, 1972.

Hayakawa, M., Itoh, T., Hattori, K., and Yumoto, K.: ULF electromagnetic precursors for an earthquake at Biak, Indonesia on 17 February 1996, Geophys. Res. Lett., 27, 1531-1534, 2000.

Huang, Q.: One possible generation mechanism of co-seismic electric signals, P. Jpn. Acad. B-Phys., 78, 173-178, 2002.

Huang, Q.: Retrospective investigation of geophysical data possibly associated with the $M_{\mathrm{S}} 8.0$ Wenchuan earthquake in Sichuan, China, J. Asian Earth Sci., 41, 421-427, 2011a.

Huang, Q.: Rethinking earthquake-related DC-ULF electromagnetic phenomena: towards a physics-based approach, Nat. Hazards Earth Syst. Sci., 11, 2941-2949, doi:10.5194/nhess-112941-2011, 2011b.
Huang, Q. and Ikeya, M.: Seismic electromagnetic signals (SEMS) explained by a simulation experiment using electromagnetic waves, Phys. Earth Planet. In., 109, 107-114, doi:10.1016/S0031-9201(98)00135-6, 1998.

Huang, Q. and Ikeya, M.: Experimental study on the propagation of seismic electromagnetic signals (SEMS) using a minigeographic model of the Taiwan strait, Episodes, 22, 289-294, 1999.

Huang, Q. and Lin, Y.: Selectivity of seismic electric signal (SES) of the 2000 Izu earthquake swarm: a 3-D FEM numerical simulation model, P. Jpn. Acad. B-Phys., 86, 257-264, doi:10.2183/pjab.86.257, 2010a.

Huang, Q. and Lin, Y.: Numerical simulation of selectivity of seismic electric signal and its possible influences, Chinese Journal Geophysics (CJG), 53, 535-543, doi:10.3969/j.issn.00015733.2010.03.007, 2010b.

Keilis-Borok, V.: The lithosphere of the Earth as a large nonlinear system, in: Quo Vadimus: Geophysics for the Next Generation, edited by: Garland, G. D. and Apel, J. R., AGU, Washington DC, Geoph. Monog. Series, 60, 81-84, 1990.

Kiratzi, A. A., Wagner, G. S., and Langston, C. A.: Source Parameters of Some Large Earthquakes in Nortern Aegean Determined by Body Waveform Inversion, Pure Appl. Geophys., 135, 515527, 1991.

Lamaignère, L., Carmona, F., and Sornette, D.: Experimental realization of critical thermal fuse rupture, Phys. Rev. Lett., 77, 2738-2741, 1996.

Nagao, T., Enomoto, Y., Fujinawa, Y., Hata, M., Hayakawa, M., Huang, Q., Izutsu, J., Kushida, Y., Maeda, K., Oike, K., Uyeda, S., and Yoshino, T.: Electromagnetic anomalies associated with 1995 Kobe earthquake, J. Geodyn., 33, 401-411, doi:10.1016/S0264-3707(02)00004-2, 2002.

Orihara, Y., Kamogawa, M., Nagao, T., and Uyeda, S.: Preseismic anomalous telluric current signals observed in Kozu-shima Island, Japan, P. Natl. Acad. Sci. USA, 109, 19125- 19128, doi:10.1073/iti4712109, 2012

Pantosti, D., De Martini, P. M., Papanastassiou, D., Palyvos, N., Lemeille, F., and Stavrakakis, G.: A Reappraisal of the 1894 Atalanti Earthquake Surface Ruptures, Central Greece, B. Seismol. Soc. Am., 91, 760-780, 2001

Ren, H., Chen, X., and Huang, Q.: Numerical simulation of coseismic electromagnetic fields associated with seismic waves due to finite faulting in porous media, Geophys. J. Int., 188, 925-944, doi:10.1111/j.1365-246X.2011.05309.x, 2012.

Rong, Y. M., Wang, Q., Ding, X., and Huang, Q. H.: Non-uniform scaling behaviour in Ultra-Low-Frequency (ULF) geomagnetic signals possibly associated with the 2011 M9.0 Tohoku earthquake, Chinese Journal Geophysics (CJG), 55, 3709-3717, 2012.

Rundle, B. J., Turcotte, D. L., and Klein, W.: Geocomplexity and the Physics of Earthquakes, American Geophysical Union, Washington DC, 2000.

Sarlis, N. V., Skordas, E. S., Lazaridou, M. S., and Varotsos, P. A.: Investigation of the seismicity after the initiation of a Seismic Electric Signal activity until the main shock, P. Jpn. Acad. BPhys., 84, 331-343, 2008.

Sarlis, N. V., Skordas, E. S., and Varotsos, P. A.: Nonextensivity and natural time: The case of seismicity, Phys. Rev. E, 82, 021110 , doi:10.1103/PhysRevE.82.021110, 2010. 
Skordas, E. S, Sarlis, N. V., and Varotsos, P. A.: Effect of significant data loss on identifying electric signals that precede rupture estimated by detrended fluctuation analysis in natural time, CHAOS, 20, 033111, doi:10.1063/1.3479402, 2010.

Sornette, A. and Sornette, D.: Earthquake rupture as a critical point: Consequences for telluric precursors, Tectonophysics, 179, 327334, 1990.

Telesca, L., Cuomo, V., and Lapenna, V.: A new approach to investigate the correlation between geoelectrical time fluctuations and earthquakes in a seismic area of southern Italy, Geophys. Res. Lett., 28, 4375-4378, 2001.

Telesca, L., Lapenna, V., and Macchiato, M.: Multifractal fluctuations in seismic interspike series, Physica A, 354, 629-640, doi:10.1016/j.physa.2005.02.053, 2005.

USGS (U.S. Geological Survey): M5.3 - 8km NW of Elatia, Greece (BETA), available at: http://comcat.cr.usgs.gov/ earthquakes/eventpage/usb000ixb7\#scientificmomenttensor, last access: 2013.

Varotsos, P. and Alexopoulos, K.: Calculation of the formation entropy of vacancies due to anharmonic effects, Phys. Rev.-B, 15, 4111-4114, 1977.

Varotsos, P. and Alexopoulos, K.: Physical properties of the variations of the electric field of the earth preceding earthquakes, I, Tectonophysics, 110, 73-98, 1984a.

Varotsos, P. and Alexopoulos, K.: Physical properties of the variations of the electric field of the earth preceding earthquakes, II. Determination of epicentre and magnitude, Tectonophysics, 110, 99-125, 1984b.
Varotsos, P. and Lazaridou, M.: Latest aspects of earthquake Prediction in Greece based on Seismic Electric Signals. I, Tectonophysics, 188, 321-347, 1991.

Varotsos, P., Alexopoulos, K., and Lazaridou, M.: Latest aspects of earthquake prediction in Greece based on Seismic Electric Signals II, Tectonophysics, 224, 1-37, 1993.

Varotsos, P., Sarlis, N., Lazaridou, M., and Kapiris, P.: Transmission of stress induced electric signals in dielectric media, J. Appl. Phys., 83, 60-70, 1998.

Varotsos, P., Sarlis, N., Skordas, E., Tanaka, H., and Lazaridou, M.: Attempt to distinguish long-range temporal correlations from the statistics of the increments by natural time analysis, Phys. Rev. E, 74, 021123, doi:10.1103/PhysRevE.74.021123, 2006.

Varotsos, P. A., Sarlis, N. V., and Skordas, E. S.: Long-range correlations in the electric signals that precede rupture, Phys. Rev. E, 66, 011902, doi:10.1103/PhysRevE.66.011902, 2002.

Varotsos, P. A., Sarlis, N. V., and Skordas, E. S.: Longrange correlations in the electric signals that precede rupture: Further investigations, Phys. Rev. E, 67, 021109, doi:10.1103/PhysRevE.78.059901, 2003.

Varotsos, P. A., Sarlis, N. V., and Skordas, E. S.: Fluctuation theorem and natural time analysis, ArXiv e-prints, 13 pp., online first, 2013.

Xu, G., Han, P., Huang, Q., Hattori, K., Febriani, F., and Yamaguchi, H.: Anomalous behaviours of geomagnetic diurnal variations prior to the 2011 off the Pacific coast Tohoku earthquake (Mw 9.0), J. Asian Earth Sci., 77, 59-65, 2013. 